\title{
PREDICTION OF INCIDENCE AND PREVALENCE OF STROKE IN NORTHEASTERN AZERBAIJAN
}

*Aliyev R.R.

Azerbaijan State Physicians' Advancement Institute named after A. Aliyev, Department of Neurologic Diseases, Baku, Azerbaijan

\begin{abstract}
The purpose of the research was studying 10 year (1999-2008) dynamics of incidence and prevalence of stroke in the northeastern region and giving a prognosis regarding the status of the incidence and prevalence of stroke in the northeast region of Azerbaijan. In this part of Azerbaijan located Guba-Khachmaz economic region. Retrospectively studied all cases of stroke in the region. 10 year chronological average level of primary incidence and prevalence of stroke were $1.2 \pm 0.05 \%$ and $2.4 \pm 0.07 \%$. It is established that the primary incidence of stroke in 1999-2008 years in the region is characterized by a tendency of low growth of disease. Predicted an increase in incidence and prevalence of stroke in 2015 - $1.56 \%$ and $3.20 \%$. Obtained data's are important for adequate therapeutic measures aimed for reducing the incidence and prevalence of stroke in the population.
\end{abstract}

Key words: stroke, incidence, prevalence, prognosis.

Literature data in 90's of last century described 9 million cases of cerebrovascular diseases annually, out of which 6 million accounted for stroke and 4.6 million of stroke cases died [1, 2, 3]. In contrast, based on WHO report for 2002, 15 million people become ill with stroke worldwide annually (mainly ischemic stroke), out of which 5 million ends lethally and another 5 million permanently become dependent on other person's help [4, 5]. Recurrent stroke occurs in $14 \%$ of stroke patients within 12 months of initial stroke [6]. Taking into account the importance of this problem, the purpose of the proposed research study was to investigate prevalence and incidence of stroke in northeast of the Azerbaijan and predict relevant values for 2015.

Material and Methods. in order to accomplish the purpose of the study, stroke incidence and prevalence data for 10-year period (1999-2008) for relevant region was analysed. Respective data was obtained from Central Regional Hospitals' Statistics Departments, city, district, and village hospitals, outpatient facilities, and physician assistant/obstetrician stations.

Number of population was determined using State Statistics Committee's National Census data [7]. Disease incidence and prevalence rates per 1000 population were calculated using variation statistics direct standardizatin. Stroke incidence and prevalence rates were predicted for the year of 2015 for the region and its individual rayons using exponential distribution method [8]. Calculations were performed using Microsoft Excel.

* e-mail: rahim_aliyev@mail.ru
Brief Summary about Region. Guba-Khachmaz economic region is situated in northeast of Azerbaijan. This region consists of Siyazan, Shabran, Guba, Gusar, and Khachmaz administrative regions. Overall area of the region is 7.66 thousand sq. km, accounting for $8.8 \%$ of country's area. This economic region is characterized with its unique landscape features. There are four sharply differing altitude zones within the region ranging from 26 meters to 4466 meters, which are plains, foothill, mid-mountaineous, and high mountaineous zones. Climate also changes depending on the altitude, as warm climatic conditions prevail in plains, whereas high mountaineous areas are characterized with cold-humid and cold climate [9]. Landscape peculiarities and climatic conditions resemble smaller model of the country.

Based on the results of National Census of Azerbaijan Republic for 2009, total number of population in the region is 488,741 thousand, accounting for $5.48 \%$ of country's population. $33.99 \%$ of population (166,105 thousand persons) reside in urban areas, whereas remaining 66.01\% (322,636 thousand persons) are settled in rural areas. The highest number of urban population is located in Siyazan $(65.6 \%)$, with the highest number of rural population being concentrated in Guba (75.11\%). $49.84 \%$ of population $(243,575$ thousand persons) consist of men and 50.16\% (245,166 thousand persons) of women. Average density of population in Guba-Khachmaz economic region is 63.8 persons per square kilometer, which is lower than country average. Average population density in 
Azerbaijan is 103.0 persons per square kilometer [7]. Depending on the landscape and climatic conditions of the region, population is mainly located in plains running along Caspian Sea [9].

Study Results. 10 year average incidence of stroke among population of Guba-Khachaz region during $1999-2008$ was $1.2 \pm 0.05 \%$. The least incidence of stroke cases in the region was noted in Siyazan in $2001(0.8 \pm 0.15 \%)$, with the highest incidence being reported in Guba in 2008 $(1.8 \pm 0.1 \%) \quad(p<0.0001)$. Lowest level of stroke incidence was reported in $1999(0.99 \pm 0.05 \%)$, with the highest level being reported in 2008 $(1.47 \pm 0.06 \%)$ (Table 1).

10 year average prevalence of stroke among population of Guba-Khachmaz economic region during 1999-2008 was $2.4 \pm 0.07 \%$. Lowest level of stroke prevalence was reported in 2001 $(2.14 \pm 0.07 \%)$, with the highest level being reported in $2008(3.0 \pm 0.09 \%)(p<0.0001)$.

10 -year average of stroke incidence among women was $1.1 \pm 0.07 \%$, whereas the same parameter was $1.2 \pm 0.07 \%$ among men $(p>0.05)$. During this time period, statistically significant difference among incidence rates of stroke for males and females was noted in 2000 only $(\mathrm{p}<0.05)$. 10year average prevalence of stroke among males and females were similar, being $2.4 \pm 0.1 \%$, with no statistically significant difference being noted for the timeframe studied $(\mathrm{p}>0.05)$.

Table 1

Stroke incidence and prevalence rates in Guba-Khachmaz region (1999-2008)

\begin{tabular}{|c|c|c|c|c|c|}
\hline \multicolumn{2}{|c|}{ Years } & \multicolumn{2}{|c|}{ Incidence } & \multicolumn{2}{c|}{ Prevalence } \\
\cline { 2 - 6 } \multicolumn{2}{|c|}{} & \%o & No & $\%$ \% & No \\
\hline \multirow{3}{*}{2999} & total & $0.99 \pm 0.05$ & 438 & $2.18 \pm 0.07$ & 968 \\
\cline { 2 - 6 } & $\mathrm{M}$ & $1.04 \pm 0.07$ & 225 & $2.16 \pm 0.1$ & 468 \\
\cline { 2 - 6 } & $\mathrm{F}$ & $0.93 \pm 0.06^{*}$ & 213 & $2.19 \pm 0.1^{*}$ & 500 \\
\hline \multirow{3}{*}{2001} & total & $1.05 \pm 0.05$ & 470 & $2.20 \pm 0.07$ & 988 \\
\cline { 2 - 6 } & $\mathrm{M}$ & $1.15 \pm 0.07$ & 252 & $2.18 \pm 0.1$ & 478 \\
\cline { 2 - 6 } & $\mathrm{F}$ & $0.95 \pm 0.07^{*}$ & 218 & $2.21 \pm 0.1^{*}$ & 510 \\
\cline { 2 - 6 } & total & $1.04 \pm 0.05$ & 471 & $2.14 \pm 0.07$ & 970 \\
\cline { 2 - 6 } & $\mathrm{F}$ & $1.10 \pm 0.07$ & 244 & $2.12 \pm 0.1$ & 469 \\
\hline \multirow{3}{*}{2002} & total & $0.98 \pm 0.07^{*}$ & 227 & $2.16 \pm 0.1^{*}$ & 501 \\
\cline { 2 - 6 } & $\mathrm{M}$ & $1.13 \pm 0.05$ & 516 & $2.25 \pm 0.07$ & 1028 \\
\cline { 2 - 6 } & $\mathrm{F}$ & $1.08 \pm 0.07$ & 265 & $2.30 \pm 0.1$ & 514 \\
\hline \multirow{3}{*}{2003} & total & $1.22 \pm 0.05$ & 251 & $2.21 \pm 0.1^{*}$ & 514 \\
\cline { 2 - 6 } & $\mathrm{M}$ & $1.25 \pm 0.08$ & 281 & $2.43 \pm 0.07$ & 1119 \\
\cline { 2 - 6 } & $\mathrm{F}$ & $1.20 \pm 0.07^{*}$ & 281 & $2.40 \pm 0.1$ & 557 \\
\hline & total & $1.12 \pm 0.05$ & 523 & $2.34 \pm 0.07$ & 562 \\
\cline { 2 - 6 } & $\mathrm{M}$ & $1.20 \pm 0.07$ & 274 & $2.32 \pm 0.1$ & 530 \\
\cline { 2 - 6 } & $\mathrm{F}$ & $1.05 \pm 0.07^{*}$ & 249 & $2.36 \pm 0.1^{*}$ & 559 \\
\hline 2005 & total & $1.16 \pm 0.05$ & 547 & $2.43 \pm 0.07$ & 1143 \\
\cline { 2 - 6 } & $\mathrm{M}$ & $1.21 \pm 0.07$ & 280 & $2.42 \pm 0.1$ & 558 \\
\cline { 2 - 6 } & $\mathrm{F}$ & $1.12 \pm 0.07^{*}$ & 267 & $2.44 \pm 0.1^{*}$ & 585 \\
\hline 2006 & total & $1.16 \pm 0.05$ & 553 & $2.41 \pm 0.07$ & 1148 \\
\cline { 2 - 6 } & $\mathrm{M}$ & $1.22 \pm 0.07$ & 287 & $2.46 \pm 0.1$ & 579 \\
\cline { 2 - 6 } & $\mathrm{F}$ & $1.10 \pm 0.07^{*}$ & 266 & $2.36 \pm 0.1^{*}$ & 569 \\
\hline 2007 & total & $1.18 \pm 0.05$ & 570 & $2.54 \pm 0.07$ & 1226 \\
\cline { 2 - 6 } & $\mathrm{M}$ & $1.21 \pm 0.07$ & 289 & $2.56 \pm 0.1$ & 610 \\
\cline { 2 - 6 } & $\mathrm{F}$ & $1.15 \pm 0.07^{*}$ & 281 & $2.52 \pm 0.1^{*}$ & 616 \\
\hline 2008 & total & $1.47 \pm 0.06$ & 714 & $3.0 \pm 0.09$ & 1460 \\
\cline { 2 - 6 } & $\mathrm{M}$ & $1.49 \pm 0.09$ & 358 & $3.05 \pm 0.1$ & 735 \\
\cline { 2 - 6 } & $\mathrm{F}$ & $1.45 \pm 0.08^{*}$ & 356 & $2.95 \pm 0.1^{*}$ & 725 \\
\hline
\end{tabular}

Note:

1. Total - total number of cases. M- males, F- females (hereinafter);

2. ${ }^{*} p>0.05, * * p<0.05 ; *$ - gender differences 
10 -year average incidence of stroke in Siyazan for the study period was $1.07 \pm 0.17 \%$. 10-year average incidence of stroke among males and females was similar, being $1.07 \pm 0.25 \%$.

Table 2 illustrates incidence and prevalence rates in Siyazan region. As seen from the table, incidence rates varied chaotically from 1999 to 2001, with a steady increase from 2001 to 2004. It remained stable in 2004-2006 and another steadily increasing trend was noted in 2006-2008. Lowest stroke incidence was noted in 2001, being $0.8 \pm 0.15 \%$ and the highest incidence rate was reported in 2008, being $1.5 \pm 0.2 \%(\mathrm{p}<0.01)$.

Comparison of stroke incidence among males and females revealed that similar values were reported in 1999-2001 and in 2006. In 2002-2004, incidence prevailed among females, whereas males became dominating in 2005, 2007, and 2008.

Stroke incidence and prevalence rates in Siyazan (1999-2008)

Table 2

\begin{tabular}{|c|c|c|c|c|c|c|}
\hline \multirow{2}{*}{\multicolumn{2}{|c|}{ Year }} & \multicolumn{2}{|c|}{ Incidence } & \multicolumn{2}{|c|}{ Prevalence } & \multirow{3}{*}{$\begin{array}{c}\begin{array}{c}\text { No. } \\
\text { population }\end{array} \\
33783\end{array}$} \\
\hline & & $\% 0$ & No & $\%$ o & No & \\
\hline \multirow{3}{*}{1999} & total & $0.9 \pm 0.2$ & 31 & $2.25 \pm 0.3$ & 76 & \\
\hline & $\mathrm{M}$ & $0.9 \pm 0.2$ & 15 & $2.0 \pm 0.4$ & 34 & 16700 \\
\hline & $\mathrm{F}$ & $0.9 \pm 0.2$ & 16 & $2.5 \pm 0.35$ & 42 & 17083 \\
\hline \multirow[t]{3}{*}{2000} & total & $1.0 \pm 0.2 *$ & 33 & $2.2 \pm 0.3^{*}$ & 74 & 34142 \\
\hline & $\mathrm{M}$ & $1.0 \pm 0.2$ & 16 & $2.0 \pm 0.3$ & 33 & 16911 \\
\hline & $\mathrm{F}$ & $1.0 \pm 0.2$ & 17 & $2.4 \pm 0.37$ & 41 & 17231 \\
\hline \multirow[t]{3}{*}{2001} & total & $0.8 \pm 0.15^{*}$ & 27 & $1.8 \pm 0.2^{*}$ & 62 & 34524 \\
\hline & $\mathrm{M}$ & $0.8 \pm 0.15$ & 14 & $1.5 \pm 0.3$ & 26 & 17132 \\
\hline & $\mathrm{F}$ & $0.8 \pm 0.15$ & 13 & $2.1 \pm 0.35$ & 36 & 17392 \\
\hline \multirow[t]{3}{*}{2002} & total & $0.9 \pm 0.2 *$ & 30 & $1.9 \pm 0.2^{*}$ & 67 & 34938 \\
\hline & $\mathrm{M}$ & $0.8 \pm 0.2$ & 13 & $1.7 \pm 0.3$ & 30 & 17347 \\
\hline & $\mathrm{F}$ & $1.0 \pm 0.2$ & 17 & $2.1 \pm 0.35$ & 37 & 17591 \\
\hline \multirow[t]{3}{*}{2003} & total & $1.0 \pm 0.2 *$ & 34 & $2.2 \pm 0.3^{*}$ & 78 & 35336 \\
\hline & $\mathrm{M}$ & $0.9 \pm 0.2$ & 15 & $2.1 \pm 0.35$ & 36 & 17570 \\
\hline & $\mathrm{F}$ & $1.1 \pm 0.25$ & 19 & $2.4 \pm 0.37$ & 42 & 17766 \\
\hline \multirow[t]{3}{*}{2004} & total & $1.1 \pm 0.2 *$ & 38 & $1.9 \pm 0.2^{*}$ & 69 & 35763 \\
\hline & M & $1.0 \pm 0.2$ & 18 & $1.7 \pm 0.3$ & 30 & 17780 \\
\hline & $\mathrm{F}$ & $1.1 \pm 0.25$ & 20 & $2.2 \pm 0.35$ & 39 & 17983 \\
\hline \multirow[t]{3}{*}{2005} & total & $1.1 \pm 0.2$ & 40 & $2.0 \pm 0.2^{*}$ & 72 & 36155 \\
\hline & $\mathrm{M}$ & $1.2 \pm 0.26$ & 21 & $1.8 \pm 0.3$ & 32 & 17999 \\
\hline & F & $1.1 \pm 0.25$ & 19 & $2.2 \pm 0.35$ & 40 & 18156 \\
\hline \multirow[t]{3}{*}{2006} & total & $1.1 \pm 0.2$ & 41 & $2.2 \pm 0.25^{*}$ & 80 & 36659 \\
\hline & M & $1.1 \pm 0.25$ & 20 & $1.9 \pm 0.3$ & 35 & 18280 \\
\hline & $F$ & $1.1 \pm 0.25$ & 21 & $2.5 \pm 0.37$ & 45 & 18379 \\
\hline \multirow[t]{3}{*}{2007} & total & $1.4 \pm 0.2 *$ & 52 & $2.2 \pm 0.2$ & 82 & 37201 \\
\hline & $\mathrm{M}$ & $1.5 \pm 0.3$ & 28 & $2.0 \pm 0.3$ & 37 & 18577 \\
\hline & $\mathrm{F}$ & $1.3 \pm 0.3$ & 24 & $2.4 \pm 0.36$ & 45 & 18624 \\
\hline \multirow[t]{3}{*}{2008} & total & $1.5 \pm 0.2 *$ & 55 & $2.2 \pm 0.2$ & 86 & 37626 \\
\hline & M & $1.55 \pm 0.3$ & 29 & $1.9 \pm 0.3$ & 38 & 18770 \\
\hline & $\mathrm{F}$ & $1.4 \pm 0.3$ & 26 & $2.5 \pm 0.36$ & 48 & 18856 \\
\hline \multirow[t]{3}{*}{ Average } & total & $1.07 \pm 0.17$ & 381 & $2.1 \pm 0.24$ & 746 & 35612.7 \\
\hline & $\mathrm{M}$ & $1.07 \pm 0.25$ & 189 & $1.87 \pm 0.3$ & 331 & 17706.6 \\
\hline & $\mathrm{F}$ & $1.07 \pm 0.25$ & 192 & $2.32 \pm 0.36$ & 415 & 17906.1 \\
\hline
\end{tabular}

Note: *p>0.05; * - in comparison with previous year

Overall average prevalence of stroke was $2.1 \pm 0.24 \%$, being $1.87 \pm 0.3 \%$ among males and $2.32 \pm 0.36 \%$ among females. Prevalence rates during the study period were higher among women compared to men. Stroke prevalence values varied randomly during these years.

10-year average of stroke incidence in Shabran during the study period was $1.0 \pm 0.1 \%$ (Table 3 ). 
Stroke incidence and prevalence in Shabran (1999-2008)

Table 3

\begin{tabular}{|c|c|c|c|c|c|c|}
\hline \multicolumn{2}{|c|}{ Year } & \multicolumn{2}{|c|}{ Incidence } & \multicolumn{2}{|c|}{ Prevalence } & \multirow{2}{*}{$\begin{array}{c}\text { No. } \\
\text { population }\end{array}$} \\
\hline & & $\% 0$ & No & $\%$ & No & \\
\hline \multirow[t]{3}{*}{1999} & total & $1.0 \pm 0.2$ & 44 & $2.1 \pm 0.2$ & 95 & 46400 \\
\hline & $\mathrm{M}$ & $1.0 \pm 0.2$ & 22 & $1.7 \pm 0.3$ & 38 & 22600 \\
\hline & $\mathrm{F}$ & $0.9 \pm 0.2$ & 22 & $2.4 \pm 0.3$ & 57 & 23800 \\
\hline \multirow[t]{3}{*}{2000} & total & $1.1 \pm 0.2^{*}$ & 50 & $2.1 \pm 0.2$ & 98 & 46800 \\
\hline & $\mathrm{M}$ & $1.1 \pm 0.2$ & 24 & $1.9 \pm 0.3$ & 43 & 22750 \\
\hline & $\mathrm{F}$ & $1.1 \pm 0.2$ & 26 & $2.3 \pm 0.3$ & 55 & 24050 \\
\hline \multirow[t]{3}{*}{2001} & total & $1.0 \pm 0.2^{*}$ & 49 & $2.0 \pm 0.2^{*}$ & 96 & 47200 \\
\hline & $\mathrm{M}$ & $1.0 \pm 0.2$ & 24 & $1.8 \pm 0.3$ & 42 & 23000 \\
\hline & $\mathrm{F}$ & $1.0 \pm 0.2$ & 25 & $2.2 \pm 0.3$ & 54 & 24200 \\
\hline \multirow[t]{3}{*}{2002} & total & $1.0 \pm 0.2$ & 49 & $2.1 \pm 0.3^{*}$ & 99 & 47700 \\
\hline & $\mathrm{M}$ & $1.0 \pm 0.2$ & 24 & $1.9 \pm 0.3$ & 43 & 23300 \\
\hline & $\mathrm{F}$ & $1.0 \pm 0.2$ & 25 & $2.3 \pm 0.3$ & 56 & 24400 \\
\hline \multirow[t]{3}{*}{2003} & total & $1.1 \pm 0.2^{*}$ & 51 & $2.2 \pm 0.2^{*}$ & 104 & 48000 \\
\hline & M & $1.1 \pm 0.2$ & 25 & $2.0 \pm 0.3$ & 47 & 23500 \\
\hline & F & $1.1 \pm 0.2$ & 26 & $2.3 \pm 0.3$ & 57 & 24500 \\
\hline \multirow[t]{3}{*}{2004} & total & $1.0 \pm 0.1^{*}$ & 50 & $2.1 \pm 0.2 *$ & 103 & 48600 \\
\hline & M & $1.0 \pm 0.2$ & 24 & $2.0 \pm 0.3$ & 47 & 23900 \\
\hline & $\mathrm{F}$ & $1.1 \pm 0.2$ & 26 & $2.3 \pm 0.3$ & 56 & 24700 \\
\hline \multirow[t]{3}{*}{2005} & total & $1.1 \pm 0.2 *$ & 51 & $2.0 \pm 0.2 *$ & 100 & 49200 \\
\hline & $M$ & $1.1 \pm 0.2$ & 26 & $1.9 \pm 0.3$ & 46 & 24200 \\
\hline & $\mathrm{F}$ & $1.0 \pm 0.2$ & 25 & $2.2 \pm 0.3$ & 54 & 25000 \\
\hline \multirow[t]{3}{*}{2006} & total & $1.0 \pm 0.1^{*}$ & 50 & $2.0 \pm 0.2$ & 101 & 49700 \\
\hline & $\mathrm{M}$ & $0.9 \pm 0.2$ & 22 & $2.0 \pm 0.3$ & 49 & 24500 \\
\hline & $\mathrm{F}$ & $1.1 \pm 0.2$ & 28 & $2.1 \pm 0.3$ & 52 & 25200 \\
\hline \multirow[t]{3}{*}{2007} & total & $1.0 \pm 0.1$ & 51 & $2.0 \pm 0.2$ & 101 & 50300 \\
\hline & $M$ & $0.9 \pm 0.2$ & 23 & $1.9 \pm 0.3$ & 48 & 24900 \\
\hline & $\mathrm{F}$ & $1.1 \pm 0.2$ & 28 & $2.1 \pm 0.3$ & 53 & 25400 \\
\hline \multirow[t]{3}{*}{2008} & total & $0.9 \pm 0.1^{*}$ & 47 & $2.0 \pm 0.2$ & 102 & 51100 \\
\hline & M & $0.8 \pm 0.2$ & 21 & $1.9 \pm 0.3$ & 47 & 25300 \\
\hline & F & $1.0 \pm 0.2$ & 26 & $2.1 \pm 0.3$ & 55 & 25800 \\
\hline \multirow[t]{3}{*}{ Average } & total & $1.0 \pm 0.1$ & 492 & $2.06 \pm 0.2$ & 999 & 48500 \\
\hline & M & $0.99 \pm 0.2$ & 235 & $1.89 \pm 0.28$ & 450 & 23795 \\
\hline & F & $1.04 \pm 0.2$ & 257 & $2.22 \pm 0.3$ & 549 & 24705 \\
\hline
\end{tabular}

Note: *p>0.05; * - in comparison with previous year

10 -year average of stroke incidence among males and females was $0.99 \pm 0.2 \%$ and $1.04 \pm 0.2 \%$, respectively. Lowest level of incidence was noted in 2008 , being $0.9 \pm 0.1 \%$, whereas the highest rate was reported in 2000, 2003 , and 2005 , being $1.1 \pm 0.2 \%$. When comparing stroke incidence among males and females, it is revealed that similar rates were observed in 200-2003, females prevailed in 2004, and 2006-2008, and males prevailed in 1999 and 2005.

Overall 10-year average prevalence of stroke was $2.06 \pm 0.2 \%$, being $22 \pm 0.3 \%$ among females and $1.89 \pm 0.28 \%$ among males. Prevalence rates were higher among females than males during the whole study period. Prevalence rates remained stable during 19992000 , being $2.1 \pm 0.2 \%$, gradually, but steadily increased in 2001-2003, steadily decreased in 2003-2005, and again remained stable in 20052008 , being $2.0 \pm 0.2 \%$.

Analysis of stroke incidence in Guba during selected study period revealed that this parameter remained stable in 1999-2001 and changed in a random manner in 2002-2008 (Table 4). 
Stroke incidence and prevalence in Guba (1999-2008)

\begin{tabular}{|c|c|c|c|c|c|c|}
\hline \multirow{2}{*}{\multicolumn{2}{|c|}{ Year }} & \multicolumn{2}{|c|}{ Incidence } & \multicolumn{2}{|c|}{ Prevalence } & \multirow{3}{*}{$\begin{array}{c}\begin{array}{c}\text { No. } \\
\text { population }\end{array} \\
136845\end{array}$} \\
\hline & & $\%$ & No & $\%$ o & No & \\
\hline \multirow[t]{3}{*}{1999} & total & $1.0 \pm 0.09$ & 133 & $2.1 \pm 0.1$ & 280 & \\
\hline & $\mathrm{M}$ & $1.1 \pm 0.1$ & 72 & $2.2 \pm 0.2$ & 149 & 66841 \\
\hline & $\mathrm{F}$ & $0.9 \pm 0.1$ & 61 & $1.9 \pm 0.2$ & 131 & 70004 \\
\hline \multirow[t]{3}{*}{2000} & total & $1.0 \pm 0.09$ & 141 & $2.1 \pm 0.1$ & 293 & 139099 \\
\hline & $\mathrm{M}$ & $1.2 \pm 0.1$ & 83 & $2.2 \pm 0.2$ & 150 & 68110 \\
\hline & $\mathrm{F}$ & $0.8 \pm 0.1$ & 58 & $2.0 \pm 0.2$ & 143 & 70989 \\
\hline \multirow[t]{3}{*}{2001} & total & $1.0 \pm 0.09$ & 131 & $2.0 \pm 0.1^{*}$ & 278 & 140000 \\
\hline & $\mathrm{M}$ & $1.1 \pm 0.1$ & 72 & $2.1 \pm 0.2$ & 147 & 68673 \\
\hline & $\mathrm{F}$ & $0.8 \pm 0.1$ & 59 & $1.8 \pm 0.2$ & 131 & 71327 \\
\hline \multirow[t]{3}{*}{2002} & total & $1.1 \pm 0.09^{*}$ & 148 & $2.2 \pm 0.1^{*}$ & 307 & 140986 \\
\hline & $\mathrm{M}$ & $1.1 \pm 0.1$ & 79 & $2.4 \pm 0.2$ & 163 & 69286 \\
\hline & $\mathrm{F}$ & $1.0 \pm 0.1$ & 69 & $2.0 \pm 0.2$ & 144 & 71700 \\
\hline \multirow[t]{3}{*}{2003} & total & $1.2 \pm 0.09 *$ & 170 & $2.3 \pm 0.1^{*}$ & 331 & 141967 \\
\hline & $\mathrm{M}$ & $1.3 \pm 0.1$ & 89 & $2.6 \pm 0.2$ & 178 & 69854 \\
\hline & F & $1.1 \pm 0.1$ & 81 & $2.1 \pm 0.2$ & 153 & 72113 \\
\hline \multirow[t]{3}{*}{2004} & total & $0.9 \pm 0.08 * *$ & 131 & $2.2 \pm 0.1^{*}$ & 310 & 143782 \\
\hline & $\mathrm{M}$ & $1.0 \pm 0.1$ & 70 & $2.3 \pm 0.2$ & 161 & 70853 \\
\hline & $\mathrm{F}$ & $0.8 \pm 0.1$ & 61 & $2.0 \pm 0.2$ & 149 & 72929 \\
\hline \multirow[t]{3}{*}{2005} & total & $1.0 \pm 0.08 *$ & 145 & $2.2 \pm 0.1$ & 323 & 145670 \\
\hline & $\mathrm{M}$ & $1.1 \pm 0.1$ & 76 & $2.4 \pm 0.2$ & 170 & 71378 \\
\hline & $\mathrm{F}$ & $0.9 \pm 0.1$ & 69 & $2.1 \pm 0.2$ & 153 & 74292 \\
\hline \multirow[t]{3}{*}{2006} & total & $1.2 \pm 0.09 *$ & 174 & $2.6 \pm 0.1 * *$ & 391 & 148489 \\
\hline & $\mathrm{M}$ & $1.3 \pm 0.1$ & 93 & $2.8 \pm 0.2$ & 209 & 73490 \\
\hline & $\mathrm{F}$ & $1.1 \pm 0.1$ & 81 & $2.4 \pm 0.2$ & 182 & 74999 \\
\hline \multirow[t]{3}{*}{2007} & total & $1.1 \pm 0.09 *$ & 169 & $2.5 \pm 0.1^{*}$ & 382 & 150600 \\
\hline & M & $1.2 \pm 0.1$ & 90 & $2.7 \pm 0.2$ & 201 & 74700 \\
\hline & $\mathrm{F}$ & $1.0 \pm 0.1$ & 79 & $2.4 \pm 0.2$ & 181 & 75900 \\
\hline \multirow[t]{3}{*}{2008} & total & $1.8 \pm 0.1 * * *$ & 279 & $3.7 \pm 0.2 * * *$ & 565 & 151900 \\
\hline & $\mathrm{M}$ & $1.9 \pm 0.2$ & 144 & $3.9 \pm 0.2$ & 294 & 75400 \\
\hline & $\mathrm{F}$ & $1.8 \pm 0.2$ & 135 & $3.5 \pm 0.2$ & 271 & 76500 \\
\hline \multirow[t]{3}{*}{ Average } & total & $1.13 \pm 0.09$ & 1621 & $2.40 \pm 0.13$ & 3460 & 143933.8 \\
\hline & $\mathrm{M}$ & $1.23 \pm 0.13$ & 868 & $2.57 \pm 0.19$ & 1822 & 70858.5 \\
\hline & $\mathrm{F}$ & $103 \pm 0.12$ & 753 & $2.24 \pm 0.18$ & 1638 & 73075.3 \\
\hline
\end{tabular}

Note: *p>0.05, ${ }^{* *} \mathrm{p}<0.05, * * * \mathrm{p}<0.0001 ; *$ - in comparison with previous year

10 -year average of stroke incidence was $1.13 \pm 0.09 \%$. 10-year average of stroke incidence among males and females were $1.23 \pm 0.13 \%$ and $1.03 \pm 0.12 \%$, respectively and did not have statistically significant differences $(p>0.05)$. However, males became ill with stroke at a higher rate than females in 2000 and $2001(\mathrm{p}<0.05)$.

The lowest incidence during the selected study period was reported in 2004 , being $0.9 \pm 0.08 \%$, whereas the highest rate was reported in 2008 , being $1.8 \pm 0.1 \%(\mathrm{p}<0.0001)$. 10-year average of stroke prevalence was $2.4 \pm 0.13 \%$. 10-year aver- age of stroke prevalence was $2.24 \pm 0.18 \%$ among females and $2.57 \pm 0.19 \%$ among males. The lowest prevalence rate of $2.0 \pm 0.1 \%$ was reported in 2001 and the highest prevalence rate of $3.7 \pm 0.2 \%$ was reported in $2008(\mathrm{p}<0.0001)$. Stroke prevalence also demonstrated random changes during studied years.

Stroke incidence during selected study period remained stable in Gusar in 1999-2001, being $1.1 \pm 0.1 \%$, peaked in 2002-2004, being $1.2 \pm 0.1 \%$, and remained almost stable in 2005-2008 (Table 5). 10-year average incidence of stroke was $1.12 \pm 0.12 \%$. 
Stroke incidence and prevalence in Gusar (1999-2008)

\begin{tabular}{|c|c|c|c|c|c|c|}
\hline \multirow{2}{*}{\multicolumn{2}{|c|}{ Year }} & \multicolumn{2}{|c|}{ Incidence } & \multicolumn{2}{|c|}{ Prevalence } & \multirow{3}{*}{$\begin{array}{c}\begin{array}{c}\text { No. } \\
\text { population }\end{array} \\
81688\end{array}$} \\
\hline & & $\%$ & No & $\%$ & No & \\
\hline \multirow[t]{3}{*}{1999} & total & $1.1 \pm 0.1$ & 90 & $2.4 \pm 0.2$ & 198 & \\
\hline & $\mathrm{M}$ & $1.2 \pm 0.2$ & 49 & $2.7 \pm 0.3$ & 108 & 39635 \\
\hline & $\mathrm{F}$ & $1.0 \pm 0.2$ & 41 & $2.1 \pm 0.2$ & 90 & 42053 \\
\hline \multirow{3}{*}{2000} & total & $1.1 \pm 0.1$ & 91 & $2.4 \pm 0.2$ & 196 & 82345 \\
\hline & $\mathrm{M}$ & $1.2 \pm 0.2$ & 49 & $2.7 \pm 0.3$ & 107 & 39915 \\
\hline & $\mathrm{F}$ & $1.0 \pm 0.2$ & 42 & $2.1 \pm 0.2$ & 89 & 42430 \\
\hline \multirow[t]{3}{*}{2001} & total & $1.1 \pm 0.1$ & 92 & $2.3 \pm 0.2 *$ & 193 & 83001 \\
\hline & $\mathrm{M}$ & $1.2 \pm 0.2$ & 50 & $2.6 \pm 0.3$ & 104 & 40568 \\
\hline & $\mathrm{F}$ & $1.0 \pm 0.2$ & 42 & $2.1 \pm 0.2$ & 89 & 42433 \\
\hline \multirow[t]{3}{*}{2002} & total & $1.2 \pm 0.1^{*}$ & 97 & $2.4 \pm 0.2 *$ & 201 & 83493 \\
\hline & M & $1.3 \pm 0.2$ & 51 & $2.7 \pm 0.3$ & 112 & 40902 \\
\hline & $\mathrm{F}$ & $1.1 \pm 0.2$ & 46 & $2.1 \pm 0.2$ & 89 & 42591 \\
\hline \multirow[t]{3}{*}{2003} & total & $1.2 \pm 0.1$ & 99 & $2.4 \pm 0.2$ & 203 & 83920 \\
\hline & $\mathrm{M}$ & $1.3 \pm 0.2$ & 52 & $2.7 \pm 0.3$ & 112 & 41212 \\
\hline & $\mathrm{F}$ & $1.1 \pm 0.2$ & 47 & $2.1 \pm 0.2$ & 91 & 42708 \\
\hline \multirow[t]{3}{*}{2004} & total & $1.2 \pm 0.1$ & 98 & $2.4 \pm 0.2$ & 199 & 84560 \\
\hline & $\mathrm{M}$ & $1.3 \pm 0.2$ & 52 & $2.6 \pm 0.3$ & 107 & 41585 \\
\hline & $\mathrm{F}$ & $1.1 \pm 0.2$ & 46 & $2.1 \pm 0.2$ & 92 & 42975 \\
\hline \multirow[t]{3}{*}{2005} & total & $1.1 \pm 0.1^{*}$ & 95 & $2.4 \pm 0.2$ & 207 & 85221 \\
\hline & $\mathrm{M}$ & $1.2 \pm 0.2$ & 51 & $2.6 \pm 0.3$ & 109 & 41994 \\
\hline & $\mathrm{F}$ & $1.0 \pm 0.2$ & 44 & $2.3 \pm 0.2$ & 98 & 43227 \\
\hline \multirow[t]{3}{*}{2006} & total & $1.1 \pm 0.1$ & 98 & $2.5 \pm 0.2 *$ & 213 & 85899 \\
\hline & $\mathrm{M}$ & $1.3 \pm 0.2$ & 53 & $2.7 \pm 0.3$ & 116 & 42440 \\
\hline & $\mathrm{F}$ & $1.0 \pm 0.2$ & 45 & $2.2 \pm 0.2$ & 97 & 43459 \\
\hline \multirow[t]{3}{*}{2007} & total & $1.0 \pm 0.1 *$ & 90 & $2.6 \pm 0.2 *$ & 229 & 86622 \\
\hline & $\mathrm{M}$ & $1.2 \pm 0.2$ & 50 & $2.8 \pm 0.3$ & 118 & 42920 \\
\hline & $\mathrm{F}$ & $0.9 \pm 0.1$ & 40 & $2.5 \pm 0.2$ & 111 & 43702 \\
\hline \multirow[t]{3}{*}{2008} & total & $1.1 \pm 0.1 *$ & 95 & $2.7 \pm 0.2 *$ & 236 & 87010 \\
\hline & $\mathrm{M}$ & $1.2 \pm 0.2$ & 52 & $2.8 \pm 0.3$ & 121 & 43100 \\
\hline & $\mathrm{F}$ & $1.0 \pm 0.2$ & 43 & $2.6 \pm 0.2$ & 115 & 43910 \\
\hline \multirow{3}{*}{ Average } & total & $1.12 \pm 0.12$ & 945 & $2.46 \pm 0.17$ & 2075 & 84375.9 \\
\hline & $\mathrm{M}$ & $1.23 \pm 0.17$ & 509 & $2.69 \pm 0.26$ & 1114 & 41427.1 \\
\hline & $\mathrm{F}$ & $1.02 \pm 0.15$ & 436 & $2.24 \pm 0.23$ & 961 & 42948.8 \\
\hline
\end{tabular}

Note: ${ }^{*} \mathrm{p}>0.05 ; *$ - in comparison with previous year

10-year average of this parameter among males and females was $1.23 \pm 0.17 \%$ and $1.02 \pm 0.15 \%$, respectively. The lowest incidence rate was noted in 2007 , being $1.0 \pm 0.1 \%$.

10 -year average prevalence of stroke in the region was $2.46 \pm 0.17 \%$. This number was $2.24 \pm 0.23 \%$ among females and $2.69 \pm 0.26 \%$ among males.

Stroke prevalence in 1999-2005 remained almost stable, being $2.4 \pm 0.2 \%(2.3 \pm 0.2 \%$ in 2001 only). 2005-2008-ci illər ərzində isə dinamik artmişdır. 10-year average of stroke incidence in Khach- males and females revealed taht the rates were maz during selected study period was $1.3 \pm 0.09 \%$ (Table 6). Relevant values for men and women were $1.28 \pm 0.13 \%$ and $1.25 \pm 0.13 \%$, respectively. The lowest incidence rate was reported in 1999 $(1.0 \pm 0.08 \%)$ and the highest rate was reported in $2008(1.5 \pm 0.1 \%) \quad(\mathrm{p}<0.0001)$. Stroke incidence steadily increased in 1999-2003, remained stable in 2003-2005 $(1.4 \pm 0.1 \%$ ), and declined in 2006 $(1.2 \pm 0.09 \%)$. In 2007 and 2008 an increasing trend was noted, the figures being $1.3 \pm 0.09 \%$ and $1.5 \pm 0.1 \%$.

Comparison of stroke incidence rates among 
Stroke incidence and prevalence in Khachmaz (1999-2008)

\begin{tabular}{|c|c|c|c|c|c|c|}
\hline \multirow{2}{*}{\multicolumn{2}{|c|}{ Years }} & \multicolumn{2}{|l|}{ Incidence } & \multicolumn{2}{|l|}{ Prevalence } & \multirow{2}{*}{$\begin{array}{l}\text { No. } \\
\text { Population }\end{array}$} \\
\hline & & $\%$ & No & $\%$ & No & \\
\hline \multirow[t]{3}{*}{1999} & total & $1.0 \pm 0.08$ & 140 & $2.2 \pm 0.1$ & 319 & 145600 \\
\hline & $M$ & $1.0 \pm 0.1$ & 67 & $2.0 \pm 0.2$ & 139 & 70500 \\
\hline & $\mathrm{F}$ & $1.0 \pm 0.1$ & 73 & $2.4 \pm 0.2$ & 180 & 75100 \\
\hline \multirow[t]{3}{*}{2000} & total & $1.1 \pm 0.09 *$ & 155 & $2.2 \pm 0.1$ & 327 & 147050 \\
\hline & $\mathrm{M}$ & $1.1 \pm 0.1$ & 80 & $2.0 \pm 0.2$ & 145 & 71150 \\
\hline & $\mathrm{F}$ & $1.0 \pm 0.1$ & 75 & $2.4 \pm 0.2$ & 182 & 75900 \\
\hline \multirow[t]{3}{*}{2001} & total & $1.2 \pm 0.09 *$ & 172 & $2.3 \pm 0.1^{*}$ & 341 & 148500 \\
\hline & $\mathrm{M}$ & $1.2 \pm 0.1$ & 84 & $2.1 \pm 0.2$ & 150 & 72200 \\
\hline & $\mathrm{F}$ & $1.2 \pm 0.1$ & 88 & $2.5 \pm 0.2$ & 191 & 76300 \\
\hline \multirow[t]{3}{*}{2002} & total & $1.3 \pm 0.09 *$ & 192 & $2.4 \pm 0.1^{*}$ & 354 & 149700 \\
\hline & $\mathrm{M}$ & $1.3 \pm 0.1$ & 98 & $2.3 \pm 0.2$ & 166 & 72900 \\
\hline & $\mathrm{F}$ & $1.3 \pm 0.1$ & 94 & $2.5 \pm 0.2$ & 188 & 76800 \\
\hline \multirow[t]{3}{*}{2003} & total & $1.4 \pm 0.1^{*}$ & 208 & $2.7 \pm 0.1 * *$ & 403 & 151000 \\
\hline & $\mathrm{M}$ & $1.4 \pm 0.1$ & 100 & $2.5 \pm 0.2$ & 184 & 73600 \\
\hline & $\mathrm{F}$ & $1.4 \pm 0.1$ & 108 & $2.8 \pm 0.2$ & 219 & 77400 \\
\hline \multirow[t]{3}{*}{2004} & total & $1.4 \pm 0.1$ & 206 & $2.7 \pm 0.1$ & 408 & 153000 \\
\hline & $\mathrm{M}$ & $1.5 \pm 0.1$ & 110 & $2.5 \pm 0.2$ & 185 & 74800 \\
\hline & $\mathrm{F}$ & $1.2 \pm 0.1$ & 96 & $2.9 \pm 0.2$ & 223 & 78200 \\
\hline \multirow[t]{3}{*}{2005} & total & $1.4 \pm 0.1$ & 216 & $2.9 \pm 0.1^{*}$ & 441 & 154400 \\
\hline & $\mathrm{M}$ & $1.4 \pm 0.1$ & 106 & $2.7 \pm 0.2$ & 201 & 75500 \\
\hline & $\mathrm{F}$ & $1.4 \pm 0.1$ & 110 & $3.0 \pm 0.2$ & 240 & 78900 \\
\hline \multirow[t]{3}{*}{2006} & total & $1.2 \pm 0.09 *$ & 190 & $2.3 \pm 0.1 * * *$ & 363 & 155800 \\
\hline & $\mathrm{M}$ & $1.3 \pm 0.1$ & 99 & $2.2 \pm 0.2$ & 170 & 76800 \\
\hline & $\mathrm{F}$ & $1.2 \pm 0.1$ & 91 & $2.4 \pm 0.2$ & 193 & 79000 \\
\hline \multirow[t]{3}{*}{2007} & total & $1.3 \pm 0.09 *$ & 208 & $2.7 \pm 0.1 * *$ & 432 & 157800 \\
\hline & $\mathrm{M}$ & $1.3 \pm 0.1$ & 98 & $2.7 \pm 0.2$ & 206 & 77200 \\
\hline & $\mathrm{F}$ & $1.4 \pm 0.1$ & 110 & $2.8 \pm 0.2$ & 226 & 80600 \\
\hline \multirow[t]{3}{*}{2008} & total & $1.5 \pm 0.1^{*}$ & 238 & $3.0 \pm 0.1 * *$ & 471 & 158800 \\
\hline & $\mathrm{M}$ & $1.4 \pm 0.1$ & 112 & $3.0 \pm 0.2$ & 235 & 78100 \\
\hline & $\mathrm{F}$ & $1.6 \pm 0.1$ & 126 & $2.9 \pm 0.2$ & 236 & 80700 \\
\hline \multirow[t]{3}{*}{ Average } & total & $1.3 \pm 0.09$ & 1925 & $2.54 \pm 0.1$ & 3859 & 152165.0 \\
\hline & $\mathrm{M}$ & $1.28 \pm 0.13$ & 954 & $2.40 \pm 0.18$ & 1781 & 74275.0 \\
\hline & $\mathrm{F}$ & $1.25 \pm 0.13$ & 971 & $2.67 \pm 0.19$ & 2078 & 77890.0 \\
\hline
\end{tabular}

Note: *p>0.05, ${ }^{* *} \mathbf{p}<0.05, * * * \mathbf{p}<0.0001 ; *$ - in comparison with previous year

similar in 1999, 2001-2003, and 2005. Females prevailed in 2007 and 2008; however, this difference was not statistically significant $(p>0.05)$. In contrast, a statistically significant difference was noted in 2004, with higher rate being reported among males $(1.5 \pm 0.1 \%$ and $1.2 \pm 0.1 \%)$ $(\mathrm{p}<0.05)$.

Discussion. Overall 10-year average of stroke prevalence was $2.54 \pm 0.1 \%$, being $2.67 \pm 0.19 \%$ and $2.4 \pm 0.18 \%$ among females and males, respectively. Average stroke incidence among females was higher in 1999-2007 compared to males. However, vice versa was noted in 2008, being
$3.0 \pm 0.2 \%$ among males and $2.9 \pm 0.2 \%$ among females. Average stroke prevalence demonstrated an increasing trend in 1999-2005, decreasing to $2.3 \pm 0.1 \%$ in 2006 and re-increasing in 2007 and 2008.

The least average incidence for 10-year study period was noted in Shabran, being $1.0 \pm 0.1 \%$, whereas the highest rate was reported in Khachmaz, being $1.3 \pm 0.09 \%(p<0.05)$. The lowest incidence rates among males and females were noted in Shabran, being $0.99 \pm 0.2 \%$ and $1.04 \pm 0.2 \%$, respectively, and the highest rates were observed in Khachmaz, 
being $1.28 \pm 0.13 \%$ and $1.25 \pm 0.13 \%$, respectively $(\mathrm{p}>0.05)$.

The lowest stroke prevalence rate in the region was noted in Shabran, being $2.06 \pm 0.2 \%$, whereas the highest rate was reported in Khachmaz, being $2.54 \pm 0.1 \%(\mathrm{p}<0.05)$. The lowest stroke prevalence rate among men in the region was reported in Siyazan $(1.87 \pm 0.3 \%)$ and the highest rate in Gusar $(2.69 \pm 0.26 \%)(\mathrm{p}<0.05)$. The lowest 10 -year average stroke prevalence rate among women was observed in Shabran $(2.22 \pm 0.3 \%)$ and the highest rate in Khachmaz $(2.67 \pm 0.19 \%)$ $(\mathrm{p}>0.05)$.

Incidence and prevalence rates for stroke in 2015 were predicted for Guba-Khachmaz economic region, as well as its administrative rayons, based on available results using exponential distribution method (Table 7). Increase in stroke incidence and prevalence has been predicted for the region for 2015.

Although, increase in stroke incidence rate was predicted for Siyazan, no difference should be observed in prevalence. Predicted values suggest that epidemiologic situation for stroke will remain stable compared to previous years in Shabran. Sharp increasing trend in both incidence and prevalence rates is being predicted for Guba, to exclude 2008. Prediction is that in Gusar, incidence rate will remain stable, with relative increase in prevalence rate being noted. In Khacmaz, it is predicted that both incidence and prevalence rates for stroke will increase.

Table 7

\section{Stroke incidence and prevalence in Guba-Khachmaz economic region (1999-2008)}

\begin{tabular}{|c|c|c|}
\hline $\begin{array}{r}\text { Epidemiologic } \\
\text { Values }\end{array}$ & Incidence (\%) & $\begin{array}{c}\text { Prevalence } \\
\text { (\%) }\end{array}$ \\
\hline Siyazan & 1.80 & 2.19 \\
\hline Shabran & 0.92 & 1.92 \\
\hline Guba & 1.71 & 3.79 \\
\hline Gusar & 1.05 & 2.81 \\
\hline Khacmaz & 1.71 & 3.39 \\
\hline Guba-Khacmaz economic region & 1.56 & 3.20 \\
\hline
\end{tabular}

Conclusion. Based on WHO reports, stroke incidence is about $2 \%$ per year [10]. This value was $1-2 \%$ in European countries in 2001-2003 [11]. Provided data suggests concluding that

\section{REFERENCES}

1. Качемаева О.В., Борисова Н.А. Эпидемиологическая характеристика инсульта в г. Уфе по данным регистра // Неврологический вестник, 2007, Т. ХХXIX, вып. 1, с. 45-48.

2. Кудряшов А.А. Анализ эпидемиологических показателей и факторов риска мозговых инсультов в популяции г. Тюмени (по данным регистра мозгового инсульта): Дис. ... канд. мед. наук. Тюмень, 2008, 161 c.

3. Тонких Р.В. Моделирование и алгоритмизация иммунометаболических процессов при цереброваскулярных заболеваниях и инсульте с прогнозированием тя- incidence and prevalence of stroke in Azerbaijan, represented by Guba-Khacmaz region, is on average level compared to other countries.

жести течения: Дис. ... док. мед. наук. Воронеж, 2009, $264 \mathrm{c}$.

4. Скворцова В.И., Евзельман М.А. Ишемический инсульт. Орел: 2006, 404 с.

5. Mackay J., Mensah G. The atlas of heart disease and stroke. Geneva: WHO, 2004 (www.who.int/cardiovascular_diseases/ resources/atlas/en).

6. Adams H. Jr, del Zoppo G., Alberts M., et al. Guidelines for the early management of adults with ischemic stroke // Stroke, 2007, v.38, p.1655-1711.

7. Azərbaycan Respublikası Dövlət Statistika Komitəsi. Azərbaycan Respublikası Ohalisinin siyahıya alınması 
2009-cu il, I hissə. Bak1: 2010, 566 s.

8. Власов В.В. Эпидемиология: Учебное пособие для вузов. Москва: Издательская группа "ГЭОТАРМедиа", 2006, 462 с.

9. Quba-Xaçmaz iqtisadi rayonu. Vikipediya. Açıq ensiklopediya. http://az.wikipedia.org/wiki/Quba-Xacmaz_iqtisadi_rayonu.

10. Суслина 3.А., Гераскина Л.А., Фонякин А.В.
Артериальная гипертония, сосудистая патология мозга и антигипертензивное лечение. Москва: Медиаграфикс, 2006, 200 с.

11. Котова Е.Ю. Клинико-эпидемиологическая характеристика, ведущие факторы риска, характер течения инсульта в г. Ульяновске (по данным Регистра инсульта): Автореферат дис. ... канд. мед. наук. Москва, 2009, 26 с

\title{
PЕЗЮМЕ
}

\section{ПРОГНОЗ ЗАБОЛЕВАЕМОСТИ И РАСПРОСТРАНЕННОСТИ ИНСУЛТА В СЕВЕРО-ВОСТОЧНОМ РЕГИОНЕ РЕСПУБЛИКИ АЗЕРБАЙДЖАН}

\author{
Алиев P.P. \\ Азербайджанский Государственный Институт Усовершенствования Врачей, \\ Баку, Азербайджан
}

Цель исследования - изучение 10-летней (1999-2008 гг.) динамики случаев заболеваемости и распространенности вследствие церебрального инсульта в северо-восточном регионе и вычисление прогноза относительно состояния эпидемиологии инсульта в отдельных регионах экономического района. В этом регионе Азербайджана находиться Куба-Хачмазский экономический район. Ретроспективно изучены все случаи инсульта в регионе. 10-летний хронологический средний уровень первичной заболеваемости инсультом составил $1,2 \pm 0,05 \%$,

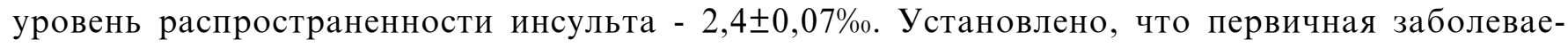
мость инсультом и его распространенность в 1999-2008 гг. в регионе характеризуется тенденцией низкого роста заболеваемости. Прогнозировано увеличение показателей заболеваемости и распространенности инсульта на 2015 год - 1,56\%о и 3,20\%. Полученные данные имеют большое значение для проведения адекватных лечебно-профилактических мероприятий, направленных на уменьшение частоты заболеваемости и смертности при инсульте среди населения.

Ключевые слова: инсульт, заболеваемость, распространенность, прогноз. 


\section{XÜLASə}

\section{AZӘRBAYCANIN ŞIMMAL-ŞəRQINDə INSULTLA XəSTӘLӘNMӘ VO ONUN YAYILMASININ PROQNOZU}

\section{Oliyev R.R.}

Ә. Oliyev ad. Azərbaycan Dövlət Həkimləri Təkmilləşdirmə Institutu, Sinir xəstəlikləri kafedrası, Bakı

Təqdim edilmiş tədqiqat işinin məqsədi respublikamızın şimal-şərqində insultla xəstələnmə və onun yayılmasının araşdırılması və bu göstəricilərin 2015-ci il üçün proqnozunun verilməsi olmuşdur. Azərbaycanın şimal-şərqində Quba-Xaçmaz iqtisadi rayonu yerləşir. Retrospektiv olaraq regionda bütün insult hadisələri öyrənilmişdir. Baş beyni insultu ilə ilkin xəstələnmənin 10 illik xronoloji orta səviyyəsi 1,2 $\pm 0,05 \%$, baş beyni insultunun yayılmasının 10 illik xronoloji orta səviyyəsi $2,4 \pm 0,07 \%$ olmuşdur. Təyin edilmişdir ki, regionda 1999-2008-ci illər ərzində baş beyni insultu ilə ilkin xəsətələnmə və onun yayılması aşağı artma tendensiyasına malikdir. Regionda 2015-ci il üçün insultla bağlı xəstələnmə, onun yayılmasının artması proqnozlaşdırılmışdır - 1,56\% və 3,20\%o. Oldə edilmiş nəticələr əhali arasında insultla bağlı xəstələnmə və ölümün azaldılmasına yönəldilmiş adekvat tibbi-profilaktik tədbirlərin aparılması üçün böyük əhəmiyyətə malikdir.

Açar sözlər: insult, xəstələnmə, yayılma proqnoz.

Redaksiyaya daxil olub: 21.01.2013

Çapa tövsiy olunub: 05.02.2013

Rəyçi: Z.M.Sadıxova, t.ü.f.d., dosent 